\title{
Interoperability Test of ebXML e-Business Solutions ${ }^{1}$
}

\author{
Peng Liang, Keqing He, Bing Li, Jin Liu \\ State Key Lab of Software Engineering, Wuhan University, \\ Wuhan 430072, P.R.China \\ liangp@sklse.org
}

\begin{abstract}
The XML-based e-Business specifications are the main stream in B2B field, and the ebXML specification is the most promising one among them with more and more e-Business solutions supporting ebXML. We argue that the interoperability assurance between different e-Business solutions is the key issue for spreading ebXML technology around the world. In this paper, we first define the concept of interoperability for software solutions, and then we propose the basic steps for Interoperability Test. At last the Interoperability Test method for ebXML solutions is presented.
\end{abstract}

Keywords: Interoperability Test, e-Business solutions, ebXML, ebMS

\section{Introduction}

Interoperability is a quite familiar and ambiguous concept in computer science. Normally we look on it in two aspects - hardware and software. The interoperability problem for software is more complex and difficult to assure than hardware. With the popularization of Internet and distributed computing, all kinds of information with different format and structure in computer system are required to be integrated seamlessly and transparently. This requirement raises the issue of interoperability. The Interoperability Assurance (IA) of e-Business and eGovernment solutions is the application of interoperability in practical domain, and standardized Interoperability Test is the most effective way to achieve the IA.

e-Business is always a hot topic in business field since its appearance with EDI system [1] in 1960s, while the high implementation cost is the biggest obstacle for SME (Small and Medium Enterprises) to come in and play [1]. With the introduction of XML (eXtensible Markup Language), the infrastructure mechanism of e-Business technology has been totally changed. The business data and process can be both specified formally in XML, and more and more XMLbased e-Business specifications appeared in the last few years. The ebXML [1] is the most promising one among them with more and more e-Business solutions supporting ebXML [5, 6]. The Interoperability Test for e-Business solutions is the process for testing how well this solution can operate and interact with other eBusiness solutions.

This paper is composed of the following sections. Section 2 describes the concept of interoperability of software solutions in details, with the basic steps of Interoperability Test. Section 3 presents the technical architecture of ebXML and its Interoperability Test levels. Section 4 presents the ebMS Interoperability Test method, including test process model and evaluation rules with a concrete example. Section 5 concludes this research with future work direction.

\section{The Interoperability}

\subsection{Interoperability of Software Solutions}

In the real enterprise computing environment, we have to face the complex system which is composed of different operating systems, databases, and applications, etc. They communicate with each other in different programming language, data structure and interface which are the common characteristics of the heterogeneous system. The IA of these heterogeneous systems is the most fundamental problem we have to solve other than security, functionality, QoS, etc. Interoperability has become the key issue for the development of information system infrastructure.

\footnotetext{
${ }^{1}$ This research project is supported by the National Natural Science Foundation of China under Grant No. 60373086; Wuhan Science \& Technique Key Project under Grant No. 20021002043; Open Foundation of SKLSE under Grant No. 03-03.
} 
The general definition of Software Interoperability is: The ability of software system to share information among different computing systems, networks, operating systems and applications. The IA of software systems can only be achieved through standardized test and certification process.

To the filed of e-Business solutions, we define the interoperability as follows: The solution can be implemented by following certain specification (e.g. ebXML) or standard, and the function of the implementation is independent from specific programming language and operating system. The implemented functionality for the interaction between solutions is checkable.

When talking about Interoperability, the Conformance issue can not be neglected. Conformance is to test whether the solution can satisfy all the requirements specified in the specification or standard, consequently the Conformance test items should be determined precisely according to the standard. The Conformance test can be conducted for a single solution. On the contrary, the Interoperability Test should be conducted between two or among more solutions, in order to test whether these solutions can complete the general function together in the real operation environment, such as the ebXML solution for business environment. The Interoperability Test items should be co-determined by domain experts and solutions vendors by referencing the standards and practical use scenario, so the Interoperability Test items are always a subset of the test items for Conformance test as shown in Figure 1.

\begin{tabular}{|c|}
\cline { 2 - 2 } \multicolumn{1}{|c|}{ Interoperability } \\
\hline Conformance \\
\hline Standard (or Specification) \\
\hline
\end{tabular}

\section{Figure 1. The Relationship among Standard, Conformance and Interoperability}

\subsection{Interoperability Test of Software Solutions}

As mentioned above, Interoperability is concerned with two or more systems, and standardization has been recognized as a key success factor of interoperability between systems. Not only the standard itself, but also the Interoperability Test process should be standardized. We summarize the basic steps of Interoperability Test as follows, and we will present detailed information in section 3 with ebXML Interoperability Test:
(1) Before performing the Interoperability Test, testers or test organization should define accurately the scope of Interoperability Test;

(2) Classify the test levels based on the test scope defined in step (1), and the lower level should be tested before the higher level;

(3) Define the detailed Interoperability Test items in each test level including the check of interoperability of datatypes, interface, syntax, semantic, process and model;

(4) Define the test process model for each test level, and define the test evaluation rules for each test item based on the test content defined in step (2) and (3).

\section{3. ebXML Interoperability Test}

The biggest advantage for applying e-Business is to find potential business partners as many as possible, not only the local partners but also partners who are located in the worldwide. Enterprise can overcome the difficulty in geography to improve the trade efficiency. Enterprises may select the e-Business solution products provided by different vendors, and the interoperability among these products is the principal problem they have to face other than security, functionality, QoS, etc.

\subsection{Technical Architecture of ebXML}

As described by OASIS, one of the designers of ebXML, "ebXML is a set of specifications, and these specifications construct the implementation framework of e-Business. The vision of ebXML is implementing a global e-Business marketplace where enterprises of any size and in any geographical location can meet and conduct business with each other through the exchange of XML-based message." [5]

As we can see, ebXML is composed of several module specifications, and each specification can be implemented independently. This condition can benefit for SME to construct its own e-Business platform gradually, and maintain the extensibility of the platform. Presently, there are 7 modules specifications in ebXML specification set as shown in Table 1 [5]:

\section{Table 1. The ebXML specifications list}

\begin{tabular}{|l|l|}
\hline Short & \multicolumn{1}{|c|}{ Full name } \\
\hline ebREQ & ebXML Requirements spec. \\
\hline ebTA & ebXML Technical Architecture spec. \\
\hline ebRIM & ebXML Registry Information Model \\
\hline ebRS & ebXML Registry Services spec. \\
\hline ebMS & ebXML Message Service spec. \\
\hline
\end{tabular}




\begin{tabular}{|l|l|}
\hline ebBPSS & ebXML Business Process spec. Schema \\
\hline ebCPPA & $\begin{array}{l}\text { ebXML Collaboration-Protocol Profile } \\
\text { and Agreement }\end{array}$ \\
\hline
\end{tabular}

\section{2. ebXML Interoperability Test Scope}

Figure 2 illustrates the typical trading scenario in ebXML: ebRIM and ebRS are used for ebXML R\&R (Registry \& Repository); ebBPSS and ebCPPA [7] are used to define the trading process and generate electronic agreement between traders; all the message communication between traders and between trader and ebXML R\&R is based on ebMS [4] specification. The ebMS specification is the fundamental part in ebXML technical architecture. It specifies the structure and function of ebXML message exchange. In this paper, we limit the Interoperability Test scope in ebMS specification.

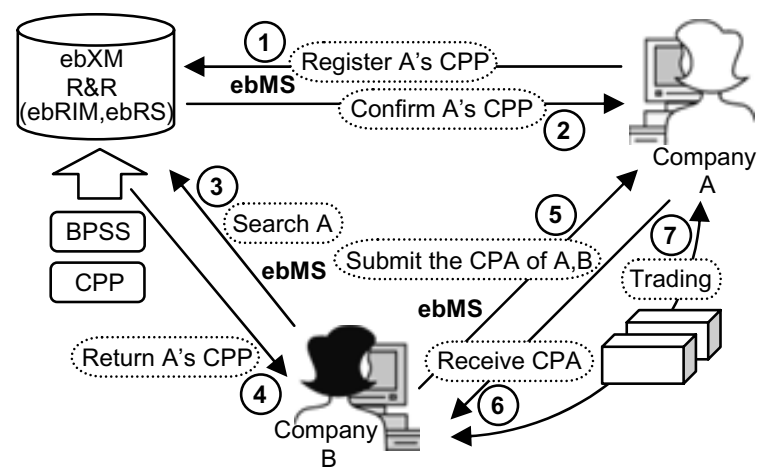

Figure 2. Typical trading scenario in ebXML

\section{3. ebMS Interoperability Test Level Classification}

As mentioned in section 2.1, the Interoperability Test items are subset of, and based on Conformance test items, meanwhile, we should consider the functional requirement in the real business when we define the Interoperability Test levels. Based on these two rules, we classify ebMS Interoperability Test levels in 2 steps: (1) Select the basic levels from ebMS 1.09 Conformance Clause [8] defined by OASIS IIC (OASIS ebXML Implementation, Interoperability and Conformance) Technical Committee; (2) Discuss with domain experts to refine the classification of general function for the real business environment;

Finally we classify the ebMS Interoperability Test items in 6 levels as shown in Table 2, and each level can test certain business function.

Table 2. ebMS interoperability test levels

\begin{tabular}{|c|l|}
\hline $\begin{array}{c}\text { Test } \\
\text { Level }\end{array}$ & \multicolumn{1}{c|}{ Description } \\
\hline T1 & One way messaging \\
\hline T2 & Synchronized message reply \\
\hline T3 & SSL security messaging \\
\hline T4 & XML signature messaging \\
\hline T5 & Reliable messaging \\
\hline T6 & Error messaging \\
\hline
\end{tabular}

\section{4. ebMS Interoperability Test Method}

\section{1. ebMS Interoperability Test Process Model}

Interoperability Test process model defines the standardized test process in order to guide the tester to perform the test in a uniform way. According to the test levels classification in section 3.3, we defined two test process models, "Single Transmission" and "Echo Back". Figure 3 illustrates the "Echo Back" test process model. In this model, message receiver should send back an acknowledge message to the sender (Step 5) when receiving a message. On the contrary, in "Single Transmission" test process model, the acknowledge message is not needed. Except that, all the other steps are the same in both "Single Transmission" and "Echo Back" test process model.

In step 1, both test parties confirm the content of the CPA file used to simulate the business context of certain test item; Step 2, sender attaches the payload file to the message, and prepare to send the message to receiver; Step 3, sender sends out the message; Step 4, receiver receives the message and extract the payload from the message. Step 6, both parties check the test result by following the test evaluation rules. In Figure 3 , the ebMS module used for ebXML Message sending/receiving is named as Message Service Handler, short for MSH.

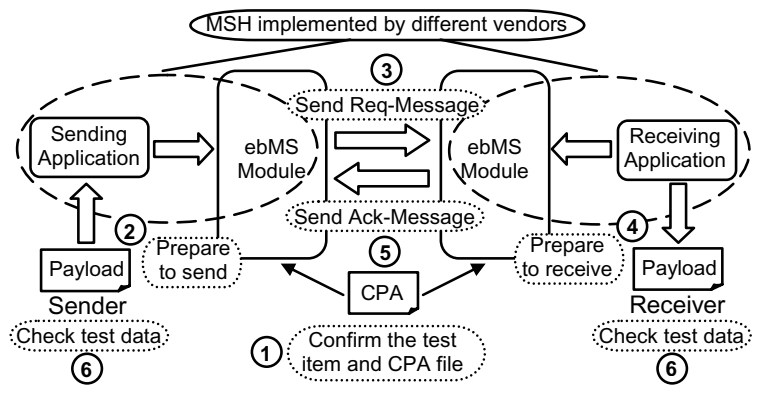

Figure 3. ebMS interoperability test process model "Echo Back" 


\section{2. ebMS Interoperability Test Evaluation Rules}

Interoperability Test evaluation rules define the judging standard for determining whether the test result satisfy the interoperability requirements of certain test item. The evaluation rule should be defined for each test item, and it includes test item general information, and test inspection items for both test parties. We demonstrate the Interoperability Test evaluation rules with a concrete example, the T1-1-1 test item in T1 level of ebMS interoperability test.

\section{Test Item General Information}

\begin{tabular}{|l|l|}
\hline Test Item ID & T1-1-1 \\
\hline Test Item Name & One way messaging \\
\hline $\begin{array}{l}\text { Test Item } \\
\text { Overview }\end{array}$ & $\begin{array}{l}\text { Sender sends a message with } \\
\text { one payload to Receiver }\end{array}$ \\
\hline CPA template & $\begin{array}{l}\text { SKLSE-CPAT111 (named and } \\
\text { prepared by test organization) }\end{array}$ \\
\hline Message Sequence & As shown in Figure 4 \\
\hline
\end{tabular}

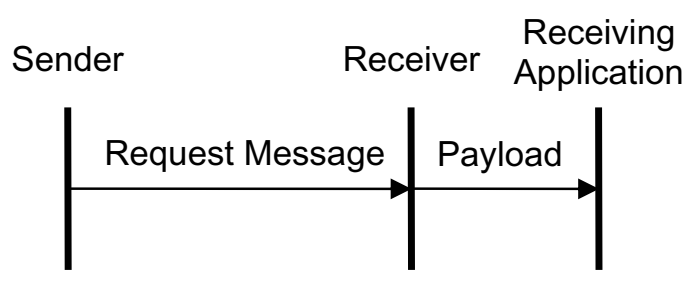

Figure 4. Message sequence of T1-1-1

\section{Test Inspection Items}

For Sender: (1) Inspect that there is no error occurred internally, no error message sent, no HTTP failure result code received.

For Receiver: (1) Inspect that there is no error occurred internally, no error message sent, no HTTP failure result code received; (2) Extract the payload in the message and compare it with the payload sent by sender, and it should be exactly the same; (3) Inspect that the value and the type attribute value of the $<$ Service $>$ element in the message is the same as defined in the CPA file; (4) Inspect that the value of the $<$ Action $>$ element in the message is the same as defined in the CPA file; (5) Inspect that the value of the $<$ CPAId $>$ element in the message is the same as defined in the CPA file.

\section{Conclusion and Future Work}

This research proposes a standard and performable Interoperability Test method for the e-Business solutions, including test process model, test evaluation rules. As a promising B2B standard, the Interoperability Test of ebXML solution has been addressed in details in this paper. The standard Interoperability Test method not only enables the vendors to produce more reliable products, but also provide decision criteria for users to select a qualified product supporting the ebXML. We argue that this Interoperability Test method can be applied not only in ebXML, but also many other XML-based e-Business specifications, such as cnXML [2], BizTalk [3] etc due to their common technical architecture. It can be also applied in the Interoperability Test between solutions based on different e-Business specifications with minor modification.

Presently the ebXML Interoperability Test is focusing on ebMS, the fundamental layer of ebXML architecture, namely the data interoperability. With the finalization of other part of ebXML specification, such as ebBPSS, ebCC, the Interoperability of business process, business dictionary, namely the process and semantic interoperability, will be considered seriously. Our research will cover these fields in the future work, and how to define the test process model in process and semantic inoperability test is the key issue.

\section{References}

[1] Alan Kotok, David R.R.Webber, ebXML: The New Global Standard for Doing Business over the Internet, New Riders, 2002.

[2] China e-Business Technology Research Center, "cnXML Overview", http://www.cnxml.org.cn/.

[3] Microsoft Corporation, "BizTalk Overview", http://www.microsoft.com/biztalk/.

[4] OASIS ebXML Technical Committee, "ebXML Message Service Specification v2.0”, 2002-04-01.

[5] OASIS ebXML Technical Committee, "ebXML Technical Architecture Specification v1.04", 2002-02-16.

[6] OASIS ebXML Technical Committee, "ebXML Requirements Specification v1.06”, 2001-05-08.

[7] OASIS ebXML Technical Committee, "ebXML Collaboration-Protocol Profile and Agreement Specification v2.0", 2002-09-23.

[8] OASIS ebXML Implementation, Interoperability and Conformance Technical Committee, "ebXML Test Framework v0.3", 2002-07-17. 